Original Article

\title{
FORMULATION AND IN VITRO EVALUATION OF GASTRO RETENTIVE IN-SITU FLOATING GELS OF TELMISARTAN CUBOSOMES
}

\author{
M. SUNITHA REDDY*, ZEBA BEGUM \\ Centre for Pharmaceutical Sciences (CPS), Institute of Science and Technology (IST), JNTUH, Kukatpally, Hyderabad 500085, Telangana \\ State, India \\ Email: baddam_sunitha@jntuh.ac.in \\ Received: 08 Sep 2021, Revised and Accepted: 11 Nov 2021
}

\begin{abstract}
Objective: Telmisartan is a BCS class II antihypertensive drug used with poor solubility. The present work aim to formulate and evaluate a sustained release formulation of Telmisartan in form of cubosomes and gastro-retentive in situ floating gels of cubosomes.

Methods: The method used was a top-down approach employing GMO(glyceryl monooleate) as a lipid phase and Pluronic F127 as a stabilizer and distilled water as aqueous phase and the resultant cubosomes dispersion is subjected to various evaluation parameters. Floating gels are prepared similarly to that of dispersion by using various gelling agents. The formulated gels are subjected to various evaluations such as pH, In vitro drug release, and kinetic release.

Results: The compatibility studies by using infrared spectroscopy showed no interaction between the drug and excipients. Cubosomes formulation TELF8 (Telmisartan cubosomes formulation) containing 40\% of GMO showed a maximum drug release of $82.75 \%$ within 20 h. This formulation exhibits an average particle size of $156.8 \mathrm{~nm}$ and a zeta potential of-30.06 mV. The concentration from the above optimized cubosome formulation is used for the preparation of floating gel using the various concentration of gelling agents(TELFNXGC)(Telmisartan gel formulation) and the sustained release was observed up to $20 \mathrm{~h}$ with a maximum drug release of about $91.58 \%$ and in vitro release kinetics exhibited sustained release
\end{abstract} follows First-order kinetics.

Conclusion: Cubosomes formulated with GMO can be used as gastro retentive sustained drug delivery vehicle and when form as a floating gel greater sustained release will be achieved.

Keywords: Cubosomes, Floating gel, Gastro-retentive

(c) 2022 The Authors. Published by Innovare Academic Sciences Pvt Ltd. This is an open access article under the CC BY license (https://creativecommons.org/licenses/by/4.0/) DOI: https://dx.doi.org/10.22159/ijcpr.2022v14i1.44111 Journal homepage: https://innovareacademics.in/journals/index.php/ijcpr

\section{INTRODUCTION}

The word cubosomes was termed by Larson, which reflects the cubic molecular crystallography similar to that of liposomes. "Cubosomes are discrete, nanostructure particles in the sub-micron range of the bicontinuous cubic liquid crystalline phase". These nanoparticles are self-organized liquid crystalline particles [1]. Cubosomes are most likely made up of polymers, lipids, and surfactants with polar and nonpolar compounds, therefore, referred to as amphiphilic [1]. Cubosomes are a bicontinuous cubic liquid phase that is surrounded by two separate water regions that are distinct by surfactantcontrolled bilayer. They are of more importance in nano-drug formulations and they can encapsulate the hydrophilic, hydrophobic, and amphiphilic compounds and the solubility of the poorly soluble drugs can be increased [2, 3]. They can be formulated by using glycerol monooleate (GMO) as the amphiphilic lipids and Pluronic as a stabilizer which can self-form into cubosomes in water [4].

Cubosomes are in general, made by high energy dispersion (sonication) of a cubic bulk phase follow by colloidal stabilization using the polymeric surfactant (top-down method) [5].

Cubosomes have advantages such as a greater surface area due to which drug payload is high, the lipids used are biodegradable and biocompatible such that hydrophilic, hydrophobic, and amphiphilic compounds can be encapsulated, the controlled release of active ingredients can be achieved and they the excellent solubilizers [6].

Glyceryl mono-oleate (GMO), a food additive approved by Food and Drug Administration, is a mixture of the glycerides of oleic acid and various fatty acids, which is mainly composed of monooleate and can form various types of lyotropic liquid crystals in the presence of water. The use of liquid crystalline phases of GMO as drug delivery systems has been extensively studied [7-9].
The model drug investigated in this study is the antihypertensive drug Telmisartan, which is used to treat Hypertension. In the present work, an attempt was made to prepare a gastro retentive sustained-release formulation of Telmisartan as cubosomes and gels, as it has an absorption window in upper GIT and a biological half-life of about $24 \mathrm{~h}$. GMO is used as the lipid phase and Pluronic F127 as a stabilizer that forms cubosomes when dispersed in water with the help of elevated energy (sonication) "GMO is also investigated as a gastro retentive lipid in some literature references, citing its bioadhesive property". The oral administration of the drug with a narrow absorption window in the gastrointestinal tract (GIT) is uniformly constrained by poor bioavailability in conventional dosage forms due to the partial drug release and the short residence time at the inclusion site. To overcome this disadvantage and to maximize the oral absorption of these drugs, new drug delivery systems have been developed. Gastro-retentive systems have been developed because they enable the controlled delivery of drugs with a prolonged residence time in the stomach of all oral dosage forms; liquid oral dosage forms are more susceptible to poor bioavailability concerning gastric drug deliveries because they are subjected to the faster passage from the stomach/duodenum. The preparation of sustained-release formulation of an oral liquid formulation could successfully be substantially improved by a liquid gel system strategy the gel formed from the in-situ gelling agents is lighter than gastric fluids, that floats above the stomach contents and creates gastric retention of the dosage form and increases gastric residence time, resulting in prolonged gastrointestinal drug delivery. The gel systems have advantages such as ease of administration, decrease administration frequency and improved patients compliances. Therefore, this research attempted to develop a formulation by combining cubosome dispersions and gels [10-14]. 


\section{MATERIALS AND METHODS}

\section{Materials}

Glyceryl monooleate (GMO) was purchased from Otto Kemi Pvt. Limited, Mumbai, India. Pluronic F127 was a gift sample from BASF. Telmisartan was a gift sample from Smilax Laboratories Hyderabad, T. S, and India. Dialysis membrane 110 was purchased from HMEDIA laboratories Hyderabad. Sodium alginate, Xanthan gum, Guar gum, and Carbopol 934 B. P were of commercial grade. The water used isdistilled water and the media used is $0.1 \mathrm{~N} \mathrm{HCl}(\mathrm{pH} 1.2)$.

\section{Equipment}

Shimadzu U. V Spectrophotometer; Bruker FTIR Spectrometer; Electronic water bath(REMI instruments); Zeta sizer (Malvern ZS 90); Diffusion cell (Locally fabricated); Ultrasonic bath Sonicator (REMI); Magnetic stirrer(REMI); pH meter(Syntronics 361); Light microscope (Edison instruments).

\section{Determination of analytical methods}

\section{Calibration curve of telmisartan}

An accurately weighed amount of $10 \mathrm{mg}$ Telmisartan was transferred into a $10 \mathrm{ml}$ volumetric flask, few drops of methanol were added to dissolve the drug then the volume was made up to the mark with methanol. From this, necessary dilutions were made to give concentrations. The absorbance of the solution was recorded at Kmax of $295 \mathrm{~nm}$ by using the Photometric method.

\section{FTIR studies}

The drug and excipients compatibility was determined by using IR spectrophotometer Bruker alpha such that there should be no interaction in between the drug and excipients and it must be compatible with each other and various peaks were obtained as follows in fig. 2.

\section{Formulation of cubosome dispersion}

Different concentrations of glyceryl monooleate (GMO) (5 to 50\%) are heated together with Pluronic F127. In an electric water bath at a temperature of 40 to $45^{\circ} \mathrm{C}$ until Pluronic F127 completely dissolves in
GMO. Telmisartan is added to the above solution and mixed well until the clear solution is obtained than add distilled water dropwise and is subjected to bath sonication for a period of 15 to $45 \mathrm{~min}$ with intermittent shaking and stirring to disperse and decompose lipid aggregates. The result is a white, opaque dispersion with no aggregates. Formulations are made in such a manner that each $5 \mathrm{ml}$ contains $20 \mathrm{mg}$ of the drug. In the case of placebo formulations, the addition of Telmisartan is skipped. The dispersions produced are stored in closed glass ampoules in a dark place at room temperature for $48 \mathrm{~h}$ and later subjected to an evaluation test.

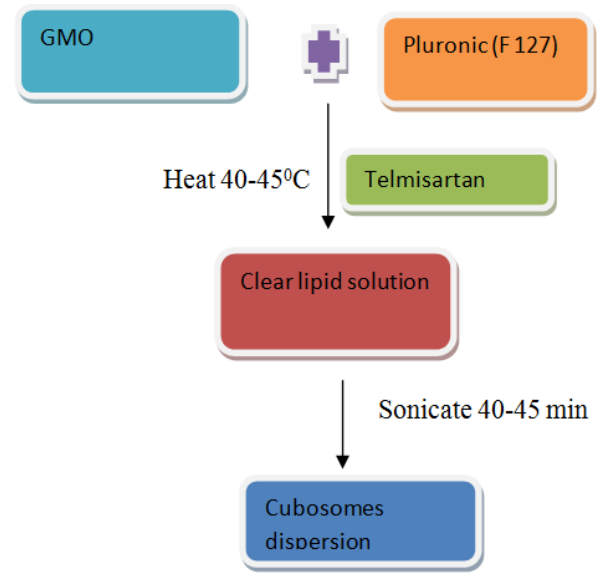

Fig. 1: Schematic representation of telmisartan cubosomes

\section{Formulation of cubosomes gels}

The floating gels are prepared similarly to cubosomes dispersion by using the optimized concentration of GMO along with Pluronic (F127) it can be used as a lipid phase and the various gelling agents such as (sodium alginate, xanthan gum, guar gum, carbopol 934 B. P) were used as an aqueous phase.

Table 1: Formulation of telmisartan-loaded cubosomes

\begin{tabular}{|c|c|c|c|c|c|}
\hline \multirow{2}{*}{ Formulation codes } & \multicolumn{2}{|c|}{ Glyceryl monooleate } & \multicolumn{2}{|c|}{ Distilled water } & \multirow{2}{*}{ Pluronic (F127) in (mg) } \\
\hline & $\% \mathrm{w} / \mathrm{v}$ & In ml & $\% \mathrm{w} / \mathrm{v}$ & In ml & \\
\hline TELF1 & 5 & 0.5 & 95 & 9.50 & 7.5 \\
\hline TELF2 & 10 & 0.25 & 90 & 9.75 & 15 \\
\hline TELF3 & 15 & 0.75 & 85 & 9.25 & 22.5 \\
\hline TELF4 & 20 & 1.00 & 80 & 9.00 & 30 \\
\hline TELF5 & 25 & 1.25 & 75 & 8.75 & 37.5 \\
\hline TELF6 & 30 & 1.50 & 70 & 8.50 & 45 \\
\hline TELF7 & 35 & 1.75 & 65 & 8.25 & 52.5 \\
\hline TELF8 & 40 & 2.00 & 60 & 8.00 & 60 \\
\hline TELF9 & 45 & 2.25 & 55 & 7.75 & 67.5 \\
\hline TELF10 & 50 & 2.50 & 50 & 7.50 & 75 \\
\hline
\end{tabular}

Table 2: Formulation of gels

\begin{tabular}{|c|c|c|c|c|c|c|}
\hline \multirow[t]{2}{*}{ Formulation code } & \multirow[t]{2}{*}{ GMO (40\%) } & \multicolumn{4}{|c|}{ An aqueous solution of gelling agents (60\%) } & \multirow{2}{*}{$\begin{array}{l}\text { Total polymer conc. } \\
(w / v)\end{array}$} \\
\hline & & Sodium alginate & Xanthan gum & Guar gum & Carbopol 934 B. P & \\
\hline TELFN1 & $"$ & 0.2 & - & - & - & 0.2 \\
\hline TELFN2 & $"$ & 0.4 & - & - & - & 0.4 \\
\hline TELFX1 & $"$ & - & 0.2 & - & - & 0.2 \\
\hline TELFX2 & $"$ & - & 0.4 & - & - & 0.4 \\
\hline TELFG1 & $"$ & - & - & 0.2 & - & 0.2 \\
\hline TELFG2 & $"$ & - & - & 0.4 & - & 0.4 \\
\hline TELFC1 & $"$ & - & - & - & 0.2 & 0.2 \\
\hline TELFC2 & $"$ & - & - & - & 0.4 & 0.4 \\
\hline TELFNX & $"$ & 0.2 & 0.2 & - & - & 0.4 \\
\hline TELFNG & $"$ & 0.2 & - & 0.2 & - & 0.4 \\
\hline TELFNXG & $"$ & 0.2 & 0.2 & 0.2 & - & 0.6 \\
\hline TELFNC & $"$ & 0.2 & - & - & 0.2 & 0.4 \\
\hline TELFNXGC & $"$ & 0.2 & 0.2 & 0.2 & 0.2 & 0.8 \\
\hline
\end{tabular}




\section{Evaluation of cubosomes dispersion}

\section{Microscopically studies}

The formulated dispersion was examined under a microscope to determine the structure of the cubosomes.

\section{Entrapment efficiency}

The Ultracentrifugation method was used to determine the \% of drug entrapped in the various formulation; the prepared samples are placed in a centrifugation tube and subjected to the instrument for centrifugation at a required speed such that the supernatant layer appears the supernatant sample is collected and examine under UV spectrophotometer such that percentage of drug entrapped can be calculated by using the formula as follow.

$\%$ Entrapment efficiency $=$ Total drug-free drug/Total drug $* 100$

\section{FTIR studies}

The interaction study of optimized formulation was evaluated by using an IR spectrophotometer; the characteristics peaks and the functional groups can be determined by the specific sharp edges of some of the IR spectrum described below.

\section{Diffusion studies}

The diffusion studies are conducted for various formulations containing the various concentration of GMO (5 to 50\%) as lipid phase and water ( 95 to $50 \%$ ) as an aqueous phase; the buffer used was $0.1 \mathrm{~N} \mathrm{HCl}$. The Franz diffusion apparatus mainly consists of two chambers one is term as donor compartment another receptor compartment the sample is placed in a donor chamber and the receptor chamber is filled with the buffer and in between the chambers the film is placed and the samples are withdrawn periodically such as $(0,1,2,3,4$, hours) and the amount of cumulative percentage drug release can be calculated.

\section{Zeta size and zeta potential determination}

The particle size and potential of the samples can be determined by using a zeta sizer (Nano SZ90, Malvern) is used; the samples are diluted in such a way that they must be clear in form and it can examine to know the size the potential.

\section{Evaluation of floating ge}

\section{Light microscope}

A light microscope (Edison Optics) is used to observe the cubosome gels at a magnification lens of 450X.

\section{Gelation property}

The Gelation property of prepared dispersions was performed by using various combinations of gelling agents such as (Sodium alginate, Xanthan gum, Guar gum, Carbopol 934B. P) and the best combination were selected, which has a good Gelation property for the further formulations.

\section{In vitro drug release}

The drug release studies were performed for the various formulations; among those, the best one is selected based on cumulative drug release and carried out for the other evaluations. The formulations of various drug release profiles and cumulative release estimates from selected gel formulations are given below.

\section{pH}

The $\mathrm{pH}$ of different concentrations of the formulation was carried out by using a $\mathrm{pH}$ meter and $\mathrm{pH}$ was recorded as follows

\section{Kinetics of drug release}

Based on drug release estimates from cubosome dispersion and gel formulation, the formulation (TELNXGC) are taken into account for release kinetics, and the conclusion is drawn from the following values by considering all the different kinetics models.

\section{RESULTS AND DISCUSSION}

\section{Calibration curve of telmisartan}

The calibration curve of telmisartan was plotted by using methanol as solvent and the Kmax was determined at $295 \mathrm{~nm}$; the different concentrations were prepared and absorbance was measured by using a spectrophotometer and the following data was obtained.

Table 3: Concentration and absorbance values

\begin{tabular}{ll}
\hline Concentration $(\boldsymbol{\mu g} / \mathbf{m l})(\mathbf{p p m})$ & Absorbance \\
\hline 2 & 0.162 \\
4 & 0.281 \\
6 & 0.460 \\
8 & 0.558 \\
10 & 0.741 \\
\hline
\end{tabular}

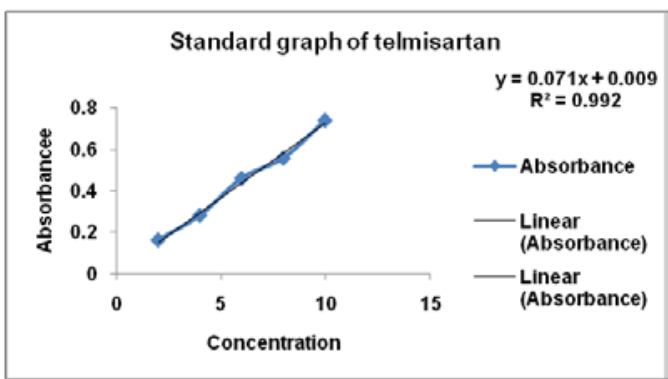

Fig. 2: Standard calibration curve of telmisartan

\section{FTIR studies}

The compatibility of drug and excipients was performed by using an IR spectrophotometer (Bruker alpha) and the following characterization peaks were observed.

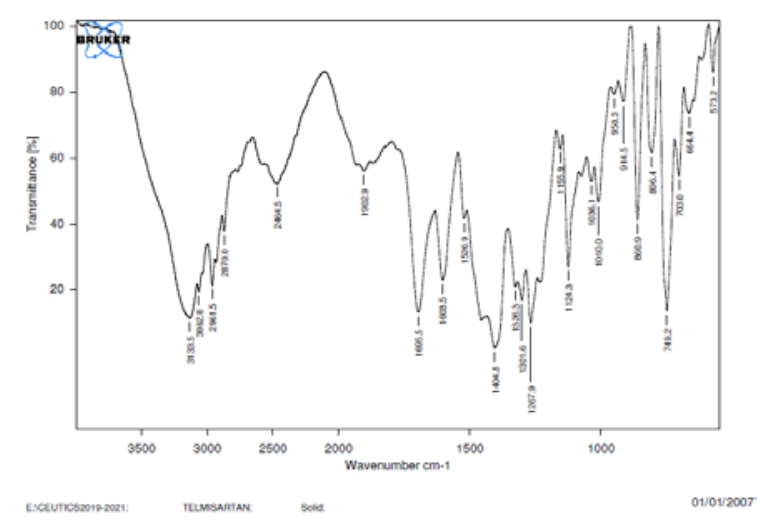

Fig. 3: IR spectrum of pure drug telmisartan 
Table 4: Frequency determining the functional groups

\begin{tabular}{lll}
\hline Frequency cm$^{-1}$ & Bond & Functional group \\
\hline $3133.5 \mathrm{~cm}^{-1}$ & O-H stretch & Alcohol \\
$2961.5 \mathrm{~cm}^{-1}$ & C-H stretch & Alkane \\
$1695.5 \mathrm{~cm}^{-1}$ & N-H stretch & Amide \\
$1124.3 \mathrm{~cm}^{-1}$ & C-O stretch & Aromatic ester \\
\hline
\end{tabular}

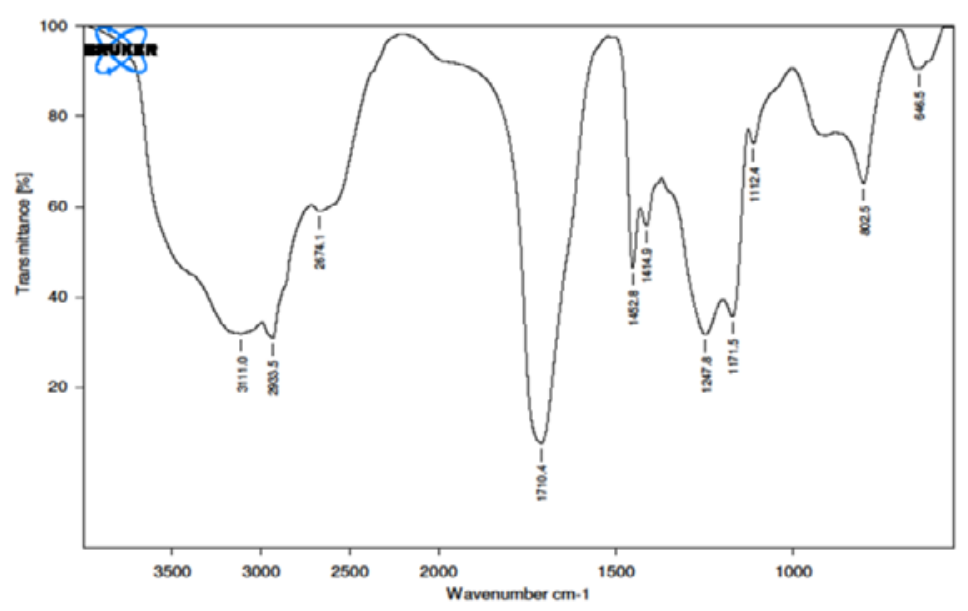

Fig. 4: IR spectrum of cubosomes dispersion

Table 5: Frequency determining the functional group

\begin{tabular}{lll}
\hline Frequency cm-1 & Bond & Functional group \\
\hline $3111.0 \mathrm{~cm}^{-1}$ & O-H stretch & Alcohol \\
$2930.5 \mathrm{~cm}^{-1}$ & C-H stretch & Alkane \\
$1710.4 \mathrm{~cm}^{-1}$ & $\mathrm{~N}-\mathrm{H}$ stretch & Amide \\
$1124.3 \mathrm{~cm}^{-1}$ & $\mathrm{C}-\mathrm{O}$ stretch & Aromatic ester \\
\hline
\end{tabular}

It was observed that the drug is compatible with all the other excipients used in the formulation and the frequency of the functional groups was found to be similar.

\section{Evaluation of telmisartan loaded cubosomes}

\section{Optical microscopy}

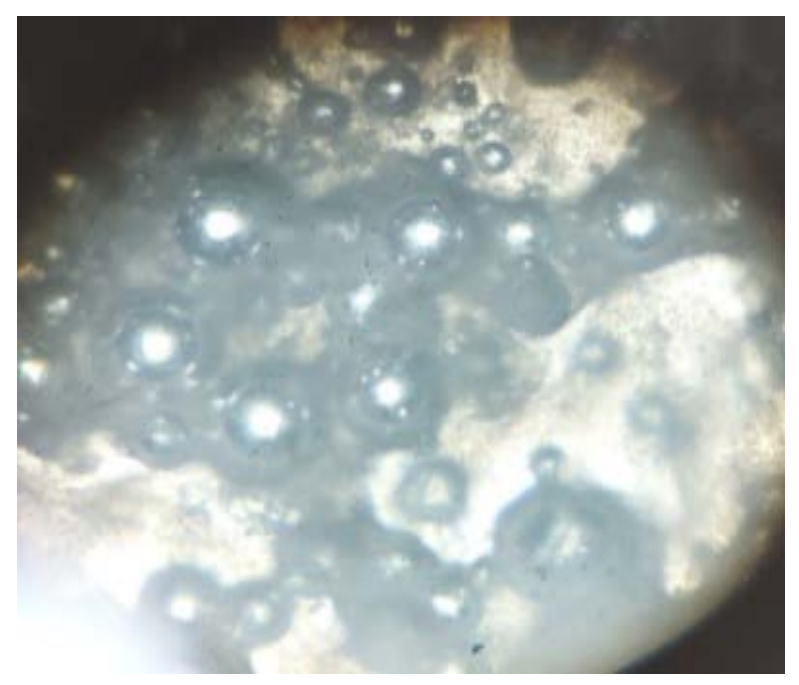

Fig. 5: Optical microscopic view

The microscopic view determines the particles are arranged in a cubic phase.
Particle size determination

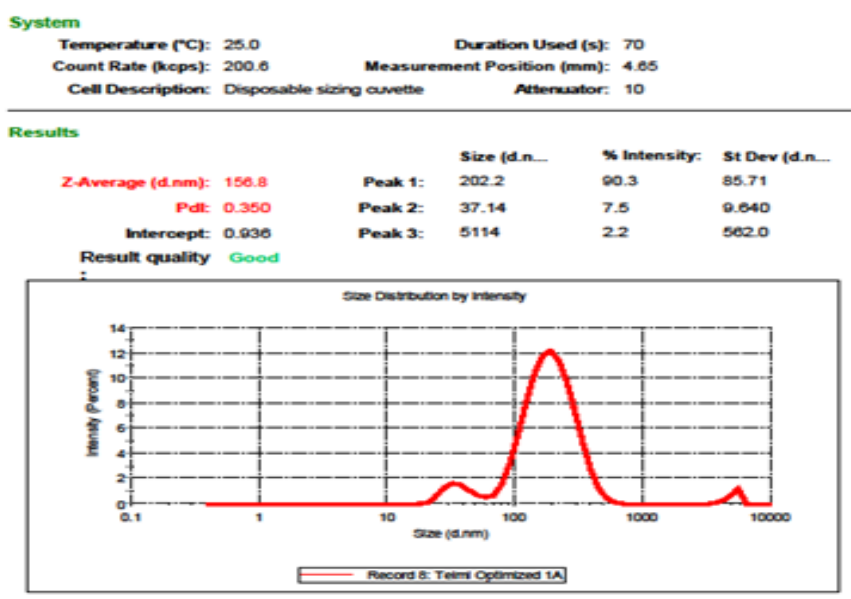

Fig. 6: Particle size of optimized formulation (TELF8)

The particle size of the optimized formulation was determined and it was found to be156 nm, which can be considered in a cubosomes range.

\section{Entrapment efficiency}

The entrapment efficiency was found to be more in the TELF8 formulation it was $93.2 \%$. 


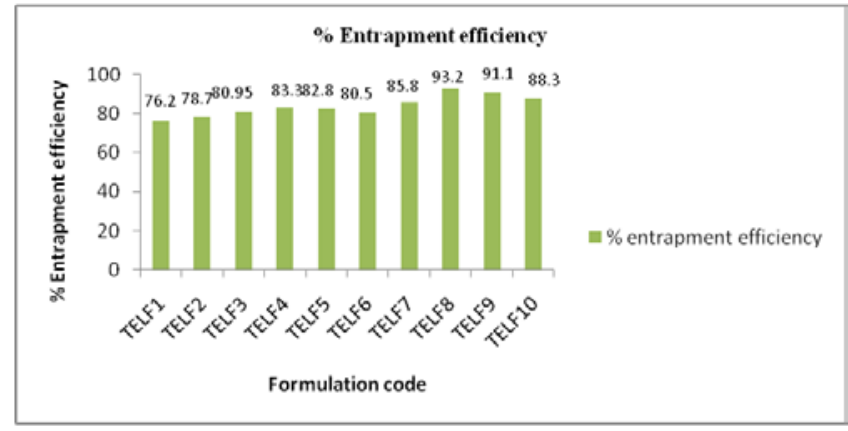

Fig. 7: Entrapment efficiency of prepared formulation

Diffusion studies of cubosomes dispersion

Table 6: Cumulative percent drug release \pm standard deviation $(n=3)$

\begin{tabular}{|c|c|c|c|c|c|c|c|c|c|c|}
\hline $\begin{array}{l}\text { Time } \\
\text { in (h) }\end{array}$ & TELF1 & TELF2 & TELF3 & TELF4 & TELF5 & TELF6 & TELF7 & TELF8 & TELF9 & TELF10 \\
\hline 0 & 0 & 0 & 0 & 0 & 0 & 0 & 0 & 0 & 0 & 0 \\
\hline 1 & $8.16 \pm 0.16$ & $10.14 \pm 0.23$ & $9.08 \pm 0.33$ & $13.10 \pm 0.18$ & $14.13 \pm 0.41$ & $15.20 \pm 0.52$ & $12.45 \pm 0.50$ & $16.52 \pm 0.21$ & $11.35 \pm 0.25$ & $9.18 \pm 0.20$ \\
\hline 2 & $10.11 \pm 0.64$ & $14.11 \pm 0.19$ & $12.14 \pm 0.62$ & $17.21 \pm 0.23$ & $20.02 \pm 0.65$ & $20.18 \pm 0.28$ & $23.29 \pm 0.59$ & $26.06 \pm 0.19$ & $14.01 \pm 1.1$ & $16.11 \pm 0.64$ \\
\hline 3 & $14.23 \pm 0.16$ & $19.29 \pm 0.17$ & $16.36 \pm 0.50$ & $21.15 \pm 0.49$ & $24.19 \pm 0.27$ & $24.39 \pm 0.39$ & $29.38 \pm 0.27$ & $32.49 \pm 0.17$ & $20.09 \pm 0.22$ & $24.09 \pm 0.72$ \\
\hline 4 & $16.28 \pm 0.21$ & $23.35 \pm 0.16$ & $24.25 \pm 0.69$ & $26.30 \pm 0.81$ & $28.32 \pm 0.47$ & $28.24 \pm 0.52$ & $32.49 \pm 0.57$ & $38.52 \pm 0.28$ & $23.16 \pm 0.29$ & $28.24 \pm 0.57$ \\
\hline 5 & $18.24 \pm 0.51$ & $26.64 \pm 0.15$ & $28.45 \pm 0.1$ & $30.45 \pm 0.38$ & $32.42 \pm 0.41$ & $33.42 \pm 0.21$ & $34.50 \pm 0.32$ & $44.59 \pm 0.28$ & $36.25 \pm 0.45$ & $32.03 \pm 0.90$ \\
\hline 6 & $20.17 \pm 0.6$ & $32.48 \pm 0.62$ & $33.74 \pm 0.21$ & $35.16 \pm 0.62$ & $36.23 \pm 0.36$ & $41.62 \pm 0.40$ & $38.17 \pm 0.85$ & $49.61 \pm 0.52$ & $38.27 \pm 0.24$ & $39.25 \pm 0.19$ \\
\hline 7 & $24.02 \pm 0.62$ & $35.52 \pm 0.27$ & $36.38 \pm 0.52$ & $38.42 \pm 0.41$ & $39.18 \pm 0.41$ & $46.06 \pm 0.59$ & $44.19 \pm 0.90$ & $52.06 \pm 0.21$ & $45.19 \pm 0.75$ & $48.60 \pm 0.32$ \\
\hline 8 & $29.12 \pm 0.27$ & $39.78 \pm 0.19$ & $40.29 \pm 0.39$ & $42.50 \pm 0.79$ & $45.09 \pm 1.2$ & $48.24 \pm 0.69$ & $49.28 \pm 0.2$ & $56.13 \pm 0.69$ & $51.17 \pm 0.26$ & $57.29 \pm 0.54$ \\
\hline 9 & $35.15 \pm 0.57$ & $43.09 \pm 0.41$ & $44.62 \pm 0.27$ & $48.19 \pm 0.17$ & $52.27 \pm 0.26$ & $51.15 \pm 0.20$ & $54.16 \pm 0.9$ & $63.26 \pm 0.72$ & $54.10 \pm 0.31$ & $58.38 \pm 0.66$ \\
\hline 10 & $39.62 \pm 0.29$ & $46.38 \pm 0.25$ & $49.61 \pm 0.49$ & $52.16 \pm 0.16$ & $56.39 \pm 0.49$ & $55.23 \pm 0.18$ & $58.09 \pm 0.62$ & $64.29 \pm 0.78$ & $58.15 \pm 0.10$ & $60.19 \pm 0.72$ \\
\hline 12 & $42.21 \pm 0.38$ & $49.32 \pm 0.39$ & $52.12 \pm 0.52$ & $56.26 \pm 0.23$ & $61.01 \pm 0.19$ & $63.36 \pm 0.33$ & $62.03 \pm 0.33$ & $66.54 \pm 0.38$ & $61.02 \pm 1.3$ & $63.16 \pm 0.9$ \\
\hline 14 & $44.04 \pm 0.64$ & $50.29 \pm 0.67$ & $56.02 \pm 0.71$ & $61.19 \pm 0.21$ & $66.14 \pm 0.10$ & $68.09 \pm 0.14$ & $69.26 \pm 0.84$ & $69.66 \pm 0.87$ & $66.16 \pm 0.29$ & $64.21 \pm 1.8$ \\
\hline 16 & $46.24 \pm 0.72$ & $53.22 \pm 0.17$ & $59.28 \pm 0.19$ & $64.08 \pm 0.20$ & $69.06 \pm 0.50$ & $70.13 \pm 0.20$ & $72.48 \pm 0.58$ & $74.29 \pm 0.67$ & $69.26 \pm 0.8$ & $68.07 \pm 0.26$ \\
\hline 18 & $48.02 \pm 0.81$ & $57.19 \pm 1.2$ & $64.16 \pm 0.2$ & $66.21 \pm 0.10$ & $72.64 \pm 0.22$ & $75.11 \pm 0.13$ & $74.29 \pm 0.56$ & $76.59 \pm 0.78$ & $72.42 \pm 0.52$ & $74.52 \pm 0.4$ \\
\hline 20 & $53.21 \pm 0.89$ & $62.01 \pm 0.25$ & $68.07 \pm 0.56$ & $69.52 \pm 0.69$ & $75.82 \pm 0.69$ & $77.29 \pm 0.11$ & $79.07 \pm 0.23$ & $82.75 \pm 0.92$ & $76.50 \pm 0.87$ & $78.09 \pm 0.33$ \\
\hline
\end{tabular}

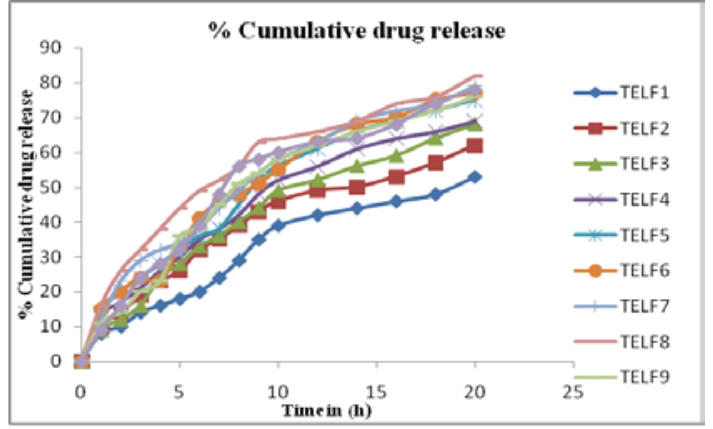

Fig. 8: \% Cumulative drug release of cubosomes dispersion

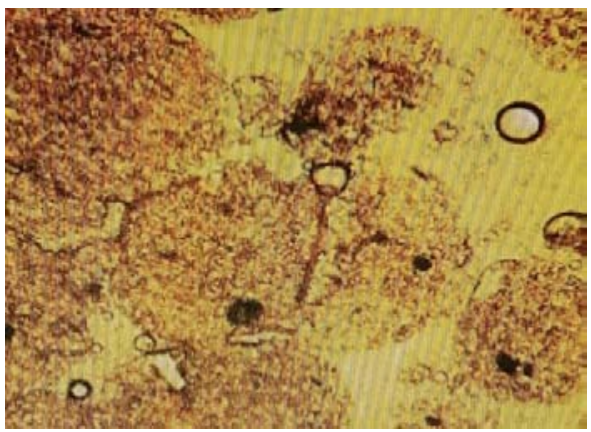

Fig. 9: Floating gel (Mag: 450X)

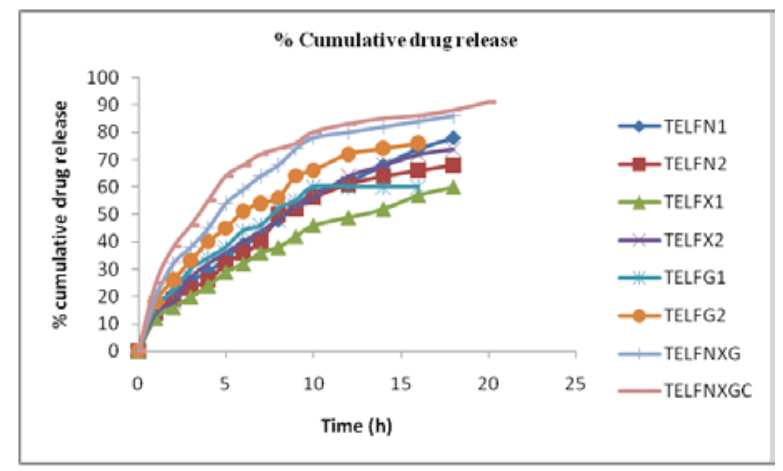

Fig. 10: \% Cumulative drug release of all combinations of gelling agents
The diffusion studies were carried out for all the formulations for the period of $20 \mathrm{~h}$, and the maximum drug release was observed in the formulation TELF8 containing 40\% of GMO the release was found to be $82.75 \%$ which can be considered the highest drug release among all the other formulation thus, it can be considered as an optimized formulation.

\section{Evaluation floating gel}

\section{Light microscope}

A light microscope (Edison Optics) is used to observe the cubosome gels at a magnification lens of 450X.

\section{Gelation property}

The combination of sodium alginate, xanthan gum, guar gum, carbopol 934 was considered the best one which provides the excellent Gelation property. 
Table 7: Gelation property of different combinations of gelling agents used in the formulation

\begin{tabular}{lll}
\hline Formulation name & Components & Results \\
\hline TELFN1 & Sodium alginate & ++ \\
TELFG1 & Guar Gum & + \\
TELFX1 & Xanthan gum & + \\
TELFC1 & Carbopol 940 & - \\
TELFNGX & Sodium alginate+Guar gum+Xanthan gum & ++ \\
TELFNGXC & Sodium alginate+Guar gum+Xanthan gum+Carbopol 934 \\
\hline
\end{tabular}

[++]: Excellent (a firm white gel will remain to foam for a long period). [+]: Good (white gel with a film-like appearance floating on the surface of $0.1 \mathrm{~N} \mathrm{HCl}$ ). [-]: Poor (gel-shaped and breaks easily on gentle agitations and shaking).

\section{In vitro drug release}

Table 8: In vitro drug release of selected gels formulations \pm standard deviation $(n=3)$

\begin{tabular}{|c|c|c|c|c|c|c|c|c|}
\hline \multirow{2}{*}{$\begin{array}{l}\text { Time in } \\
\text { (H) }\end{array}$} & \multicolumn{8}{|c|}{$\%$ Cumulative drug release } \\
\hline & TELFN1 & TELFN2 & TELFX1 & TELFX2 & TELFG1 & TELFG2 & TELFNXG & TELFNXC \\
\hline 0 & 0 & 0 & 0 & 0 & 0 & 0 & 0 & 0 \\
\hline 1 & $16.42 \pm 0.3$ & $14.12 \pm 0.9$ & $12.02 \pm 0.4$ & $14.22 \pm 0.1$ & $16.30 \pm 1.3$ & $18.09 \pm 0.6$ & $20.25 \pm 0.2$ & $25.47 \pm 0.9$ \\
\hline 2 & $21.20 \pm 1.2$ & $19.06 \pm 0.6$ & $16.51 \pm 0.1$ & $18.32 \pm 1.2$ & $22.46 \pm 0.8$ & $26.25 \pm 0.2$ & $32.16 \pm 0.4$ & $38.21 \pm 0.4$ \\
\hline 3 & $26.33 \pm 0.6$ & $23.45 \pm 0.7$ & $20.19 \pm 0.8$ & $27.20 \pm 0.9$ & $30.21 \pm 0.2$ & $33.17 \pm 0.3$ & $38.29 \pm 0.1$ & $46.35 \pm 0.7$ \\
\hline 4 & $29.45 \pm 0.9$ & $26.01 \pm 0.3$ & $24.77 \pm 0.2$ & $32.49 \pm 0.7$ & $34.62 \pm 0.1$ & $40.10 \pm 0.5$ & $45.11 \pm 0.1$ & $55.82 \pm 1.1$ \\
\hline 5 & $34.10 \pm 0.1$ & $32.14 \pm 1.1$ & $29.65 \pm 0.7$ & $36.26 \pm 0.3$ & $38.32 \pm 0.8$ & $45.24 \pm 0.7$ & $54.41 \pm 1.1$ & $64.09 \pm 1.5$ \\
\hline 6 & $39.20 \pm 0.8$ & $36.08 \pm 0.1$ & $32.23 \pm 0.4$ & $40.15 \pm 0.5$ & $44.69 \pm 0.7$ & $51.87 \pm 0.8$ & $59.67 \pm 0.9$ & $68.74 \pm 1.9$ \\
\hline 7 & $42.51 \pm 0.4$ & $40.20 \pm 0.3$ & $36.82 \pm 0.2$ & $44.72 \pm 1.2$ & $46.72 \pm 0.3$ & $54.80 \pm 0.5$ & $64.27 \pm 0.8$ & $72.27 \pm 0.2$ \\
\hline 8 & $48.11 \pm 1.1$ & $50.35 \pm 0.5$ & $38.57 \pm 0.3$ & $48.79 \pm 0.8$ & $52.45 \pm 0.4$ & $56.59 \pm 0.3$ & $68.59 \pm 0.7$ & $74.35 \pm 0.7$ \\
\hline 9 & $53.09 \pm 0.9$ & $52.79 \pm 0.8$ & $42.33 \pm 0.2$ & $53.41 \pm 1.7$ & $55.49 \pm 0.5$ & $64.06 \pm 0.9$ & $74.67 \pm 0.4$ & $76.65 \pm 1.1$ \\
\hline 10 & $58.26 \pm 0.5$ & $56.62 \pm 0.2$ & $46.58 \pm 0.7$ & $56.06 \pm 0.2$ & $60.84 \pm 0.1$ & $66.78 \pm 1.6$ & $78.20 \pm 0.3$ & $80.28 \pm 0.5$ \\
\hline 12 & $62.79 \pm 0.2$ & $61.57 \pm 0.7$ & $49.28 \pm 0.4$ & $64.15 \pm 0.1$ & $62.54 \pm 0.3$ & $72.28 \pm 0.7$ & $80.01 \pm 0.6$ & $83.19 \pm 1.8$ \\
\hline 14 & $68.06 \pm 0.1$ & $64.48 \pm 0.4$ & $52.11 \pm 0.9$ & $68.21 \pm 0.4$ & $64.29 \pm 0.8$ & $74.45 \pm 0.5$ & $82.12 \pm 1.6$ & $85.06 \pm 0.9$ \\
\hline 16 & $74.24 \pm 0.9$ & $66.27 \pm 0.3$ & $57.09 \pm 0.5$ & $72.02 \pm 0.3$ & $66.30 \pm 0.9$ & $76.36 \pm 0.3$ & $84.07 \pm 0.2$ & $86.12 \pm 0.1$ \\
\hline 18 & $78.83 \pm 0.3$ & $68.02 \pm 0.8$ & $60.82 \pm 0.6$ & $74.24 \pm 0.1$ & - & - & $86.75 \pm 0.1$ & $88.92 \pm 0.3$ \\
\hline 20 & - & - & - & - & - & - & - & $91.58 \pm 0.7$ \\
\hline
\end{tabular}

Table 9: Time and cumulative drug release of all evaluated formulation

\begin{tabular}{lll}
\hline Formulation code & \% cumulative drug release & Sustain-release duration (h) \\
\hline TELFN1 & 78 & 18 \\
TELFN2 & 68 & 18 \\
TELFX1 & 60 & 18 \\
TELFX2 & 74 & 18 \\
TELFG1 & 60 & 16 \\
TELFG2 & 76 & 16 \\
TELFNXG & 86 & 18 \\
TELFNXGC & 91 & 20 \\
\hline
\end{tabular}

The drug release was carried out among all the formulations; the drug release was maximum was found in the TELFNXGC formulation it was for about 20 h with $91.58 \%$

pH

Table 10: pH of optimized gel formulations

\begin{tabular}{lll}
\hline S. No. & Formulation code & pH \\
\hline 1 & TELF8 & 6.81 \\
2 & TELFN1 & 6.74 \\
3 & TELFN2 & 6.78 \\
4 & TELFX1 & 6.65 \\
5 & TELFX2 & 6.52 \\
6 & TELFG1 & 6.80 \\
7 & TELFG2 & 6.76 \\
8 & TELFNCG & 6.40 \\
9 & TELFNXGC & 6.68 \\
\hline
\end{tabular}




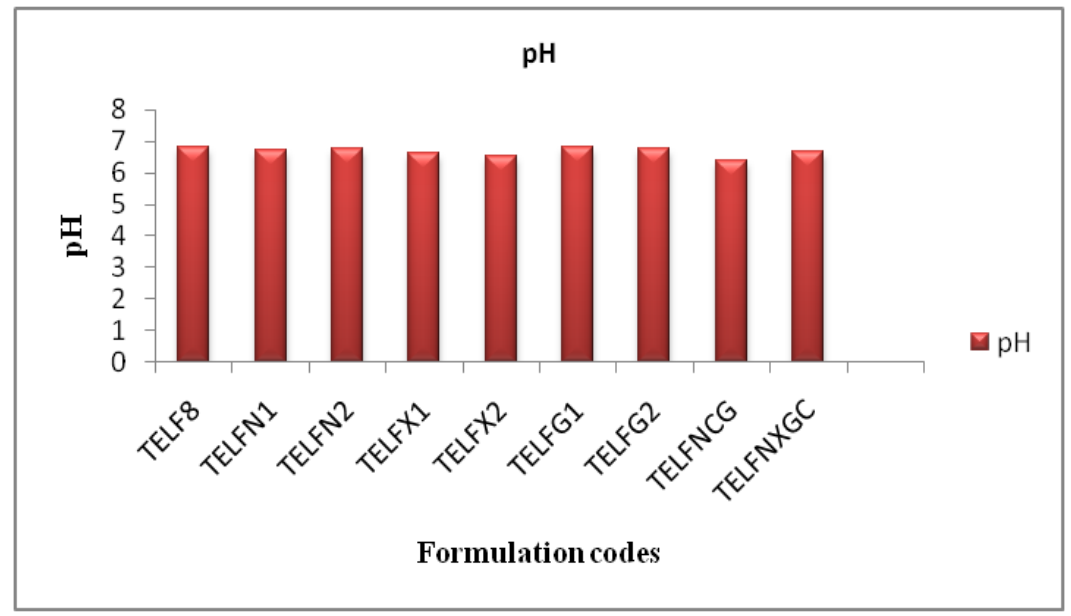

Fig. 11: pH of the optimized gel formulation

The determination of $\mathrm{pH}$ was carried out by using a pH meter; the $\mathrm{pH}$ values were obtained were under the required range.

\section{Kinetics of drug release}

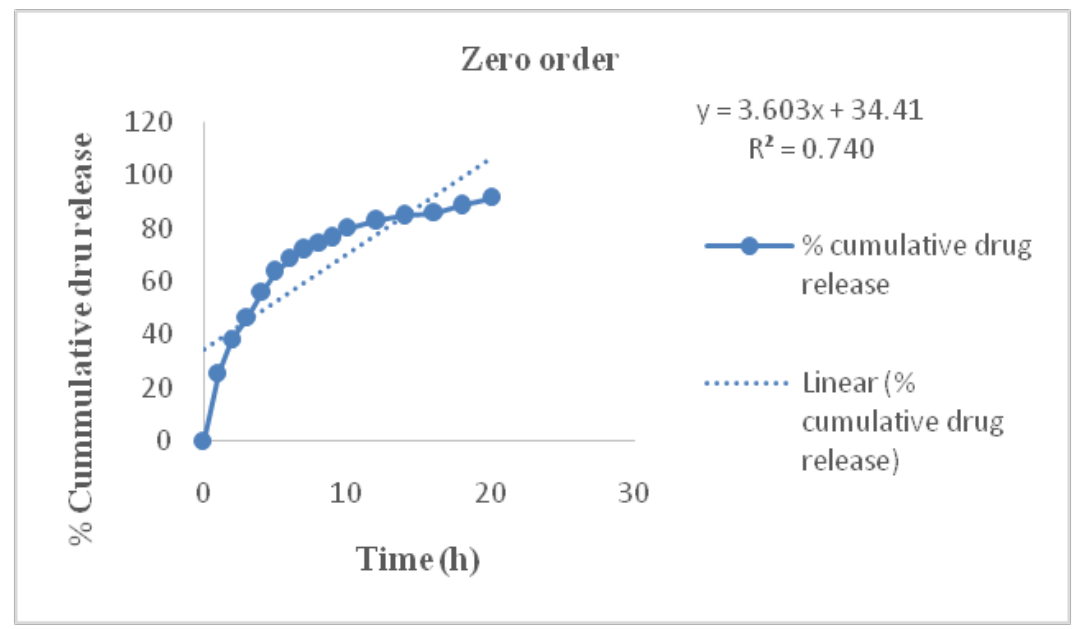

Fig. 12: Zero-order kinetics

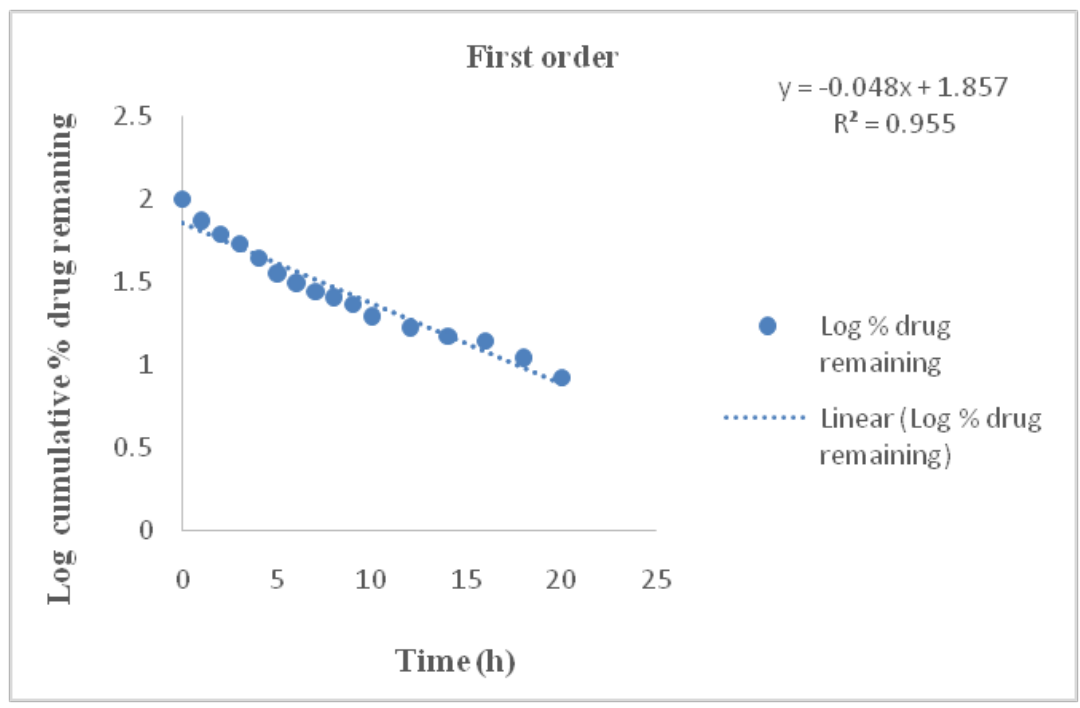

Fig. 13: First order kinetics 


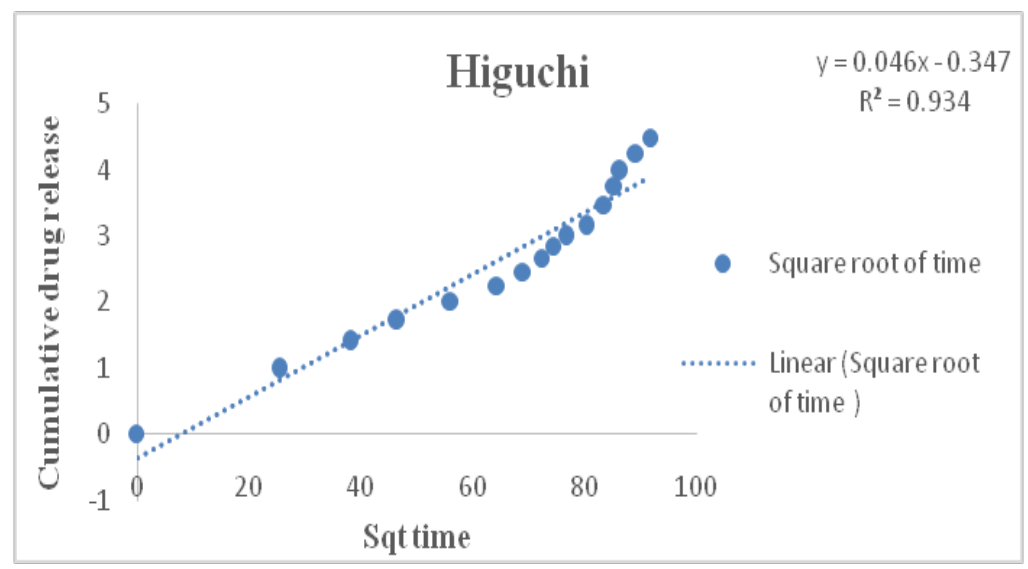

Fig. 14: Higuchi model

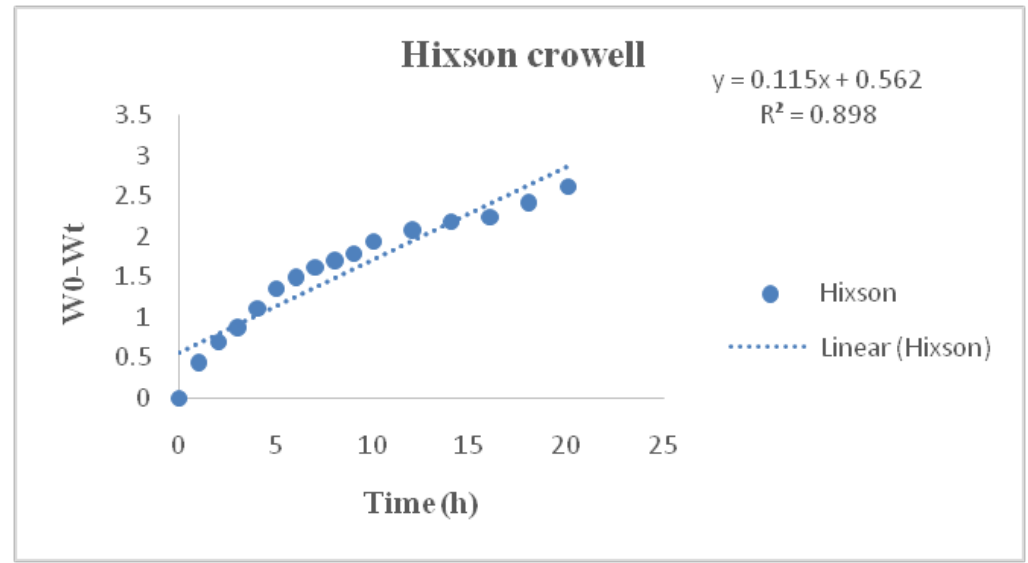

Fig. 15: Hixson crowell model

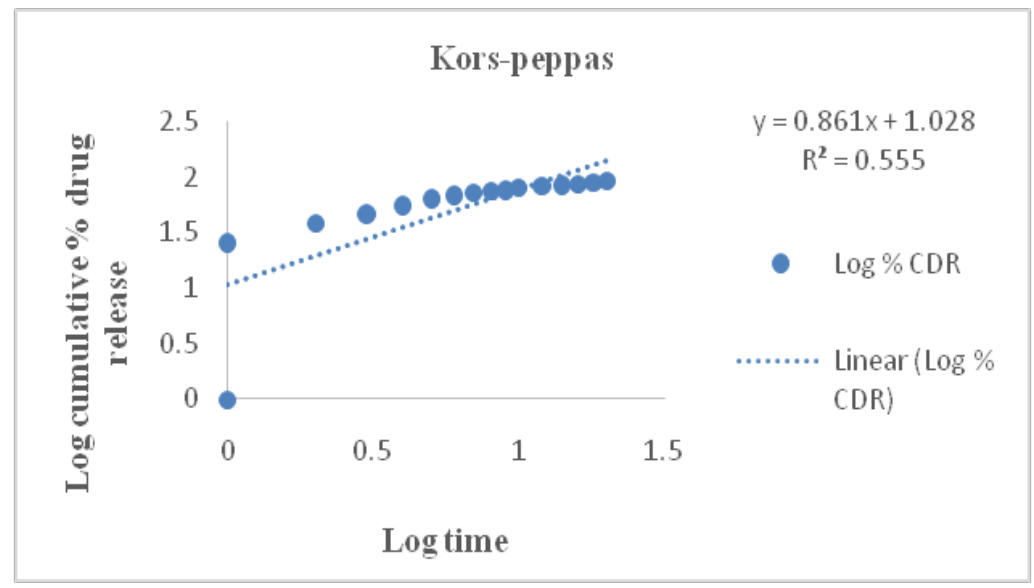

Fig. 16: Kors-peppas model

Table 11: Release kinetics of optimized gel formulation (TELFNXGC)

\begin{tabular}{ll}
\hline Kinetics model & $\mathbf{r}^{2}$ value \\
\hline Zero-order & 0.740 \\
First-order & 0.955 \\
Higuchi & 0.934 \\
Korsmeyer-Peppas kinetics & 0.555 \\
Hixson Crowell kinetics & 0.898 \\
\hline
\end{tabular}

Based on the above results, the cubosomes dispersion floating gel follows First order release kinetics. 


\section{Stability studies}

The stability studies were carried out as per ICH guidelines by considering the Accelerated stability studies were carried out by considering the temperature and relative humidity $40 \pm 2{ }^{\circ} \mathrm{C} / 75 \pm 5 \%$ $\mathrm{RH}$.

The $\mathrm{pH}$ and drug release were performed as follows.

pH

Table 12: pH of optimized formulation (TELFNXGC)

\begin{tabular}{ll}
\hline Times in days & pH of TELFNXGC \\
\hline 0 & 6.68 \\
30 & 6.42 \\
60 & 6.38 \\
90 & 6.23 \\
\hline
\end{tabular}

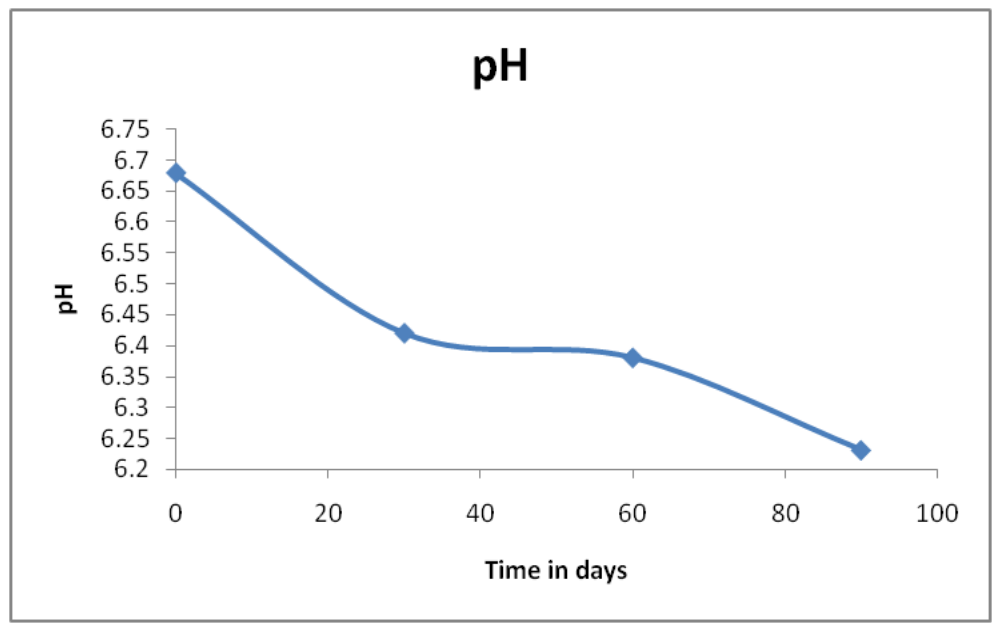

Fig. 17: pH of optimized formulation (TELFNXGC)

\section{Stability studies of drug release}

Table 13: Stability studies of optimized formulation (TELFNXGC) value in the mean of cumulative \% drug release \pm standard deviation ( $=3$ )

\begin{tabular}{llll}
\hline \% Drug release & Time in days & & \\
\cline { 2 - 4 } & $\mathbf{1}$ & $\mathbf{3 0}$ & $\mathbf{6 0}$ \\
\hline TELFNXGC & $91.68 \pm 0.63$ & $91.48 \pm 0.50$ & $91.12 \pm 0.29$
\end{tabular}

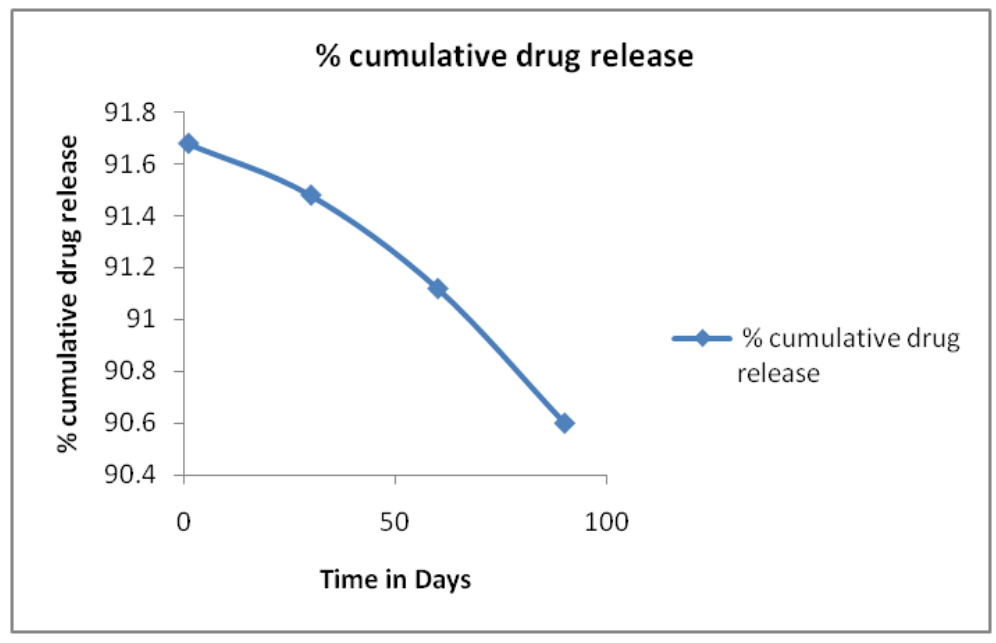

Fig. 18: Stability studies of optimized formulation (TELFNXGC)

Slight changes are observed which correspond to the stability of the selected optimized formulation (TELFNXGC). 


\section{Summary and conclusion}

The main reason for the study is gastrointestinal retention. Although many gastro-retentive formulations have been developed, this formulation deserves attention due to its unique liquid crystalline structure and its ease of preparation. Its ease of preparation. Advantages such as a high degree of biocompatibility of GMO, the ability to absorb different drugs regardless of their hydrophilic or hydrophobic nature, the effect sustained-release leads to the study of the formulation of liquid crystalline drug delivery via different routes of administration. Cubosomes are produced by GMO, which serve as a lipid when it is added to water since it tends to separate in the aqueous phase; Pluronic F127 is used as a stabilizer to prevent aggregation. The model drug Telmisartan is a BCS class 2 antihypertensive drug that, with poor solubility has an absorption window in the upper GIT. The cubosomes formulation prepared TELF8 above shows a satisfactory drug release pattern it was about $82.75 \%$ which has been selected as optimized formulation and it was used for the formulation of floating gel by using various gelling agents such as sodium alginate, xanthan gum, guar gum, carbopol the TELFNXGC shows a maximum drug release of about $91.68 \%$ for about $20 \mathrm{~h}$ according to First-order Kinetics the formulation has a delay time in fractions of a second and the formed floating gel immediately after contact with $0.1 \mathrm{~N} \mathrm{HCl}$ that likely is"Mechanism of gel formulation is the $\mathrm{pH}$-dependent cross-linking of the gelling agent surrounding the cubosomes". The above research specifies the cubosomal usefulness as a controlled release drug carrier. Extended gastric retention is achieved when they are formulated as gels that retain the cubosome structure. Although they have beneficial properties, there is still a long way to go before they can be used in clinical practice. We hope that in the near future await will be opened up so that such formulation can be used as a novel drug delivery system to overcome the disadvantages of conventional dosage forms.

\section{ACKNOWLEDGMENT}

I would like to sincerely thank Dr. M. Sunitha Reddy for her valuable guidance throughout the project and also the Center of Pharmaceutical Sciences, Institute of Science and Technology, JNTU $\mathrm{H}$ ) for providing suitable facilities to carry on the above research.

\section{FUNDING}

Nil

\section{AUTHORS CONTRIBUTIONS}

All the authors have contributed equally.

\section{CONFLICT OF INTERESTS}

Declared none

\section{REFERENCES}

1. Sadhu VR, Beram NS, Kantamneni P. A review on cubosome: the novel drug delivery system. GSC Biol Pharm Sci 2018;5(1):76-81. doi: 10.30574/gscbps.2018.5.1.0089.
2. Dhadwal A, Sharma DR, Pandit V, Ashawat MS, Kumar P. Cubosomes: A novel Carrier for transdermal drug delivery. J Drug Delivery Ther. 2020;10(1):123-30. doi: 10.22270/jddt.v10i1.3814.

3. Ramya Sri V, Madhusudhna Reddy A, Karthikeyan R, Srivinasababu P. A review on cubosomes drug delivery system. Indian J Drugs. 2017;5(3):104-8.

4. Gaballa S, El Garhy O, Abdelkader H. Cubosomes: composition, preparation, and drug delivery applications. J Adv Biomed Pharm Sci. 2019. doi: 10.21608/jabps.2019.16887.1057.

5. Naveen S, Taj Indria Muzib. A review on liquid crystalline nanoparticles (Cubosomes) emerging nanoparticulate drug carrier. Int J Curr Pharm Res. 2019;12(1):5-9.

6. Lai Jie, Chen Jianming, Lu Y, Sun Jing, Hu Fuqiang, Yin Zongning, Wu Wei. Glyceryl monooleate/poloxamer 407 cubic nanoparticles as oral drug delivery systems: I. In vitro evaluation and enhanced oral bioavailability of the poorly water-soluble drug simvastatin. AAPS PharmSciTech. 2009;10(3):960-6. doi: 10.1208/s12249-009-9292-4, PMID 19636709.

7. Tamayo Esquivel D, Ganem Quintanar A, Martinez AL, Navarrete Rodriguez M, Rodriguez Romo S, Quintanar Guerrero D. Evaluation of the enhanced oral effect of omapatrilat-monolein nanoparticles prepared by the emulsification-diffusion method. J Nanosci Nanotechnol. 2006;6(9-10):3134-8. doi: 10.1166/jnn.2006.474, PMID 17048528.

8. Leesajakul Warunee, Nakano Minoru, Taniguchi Atsuhiko, Handa Tetsurou. Interaction of cubosomes with plasma components resulting in the destabilization of cubosomes in plasma. Colloids Surf B Biointerfaces. 2004;34(4):253-8. doi: 10.1016/j.colsurfb.2004.01.010, PMID 15261065.

9. Gan L, Han Shun, Shen Jinqiu, Zhu Jiabi, Zhu Chunliu, Zhang Xinxin, Gan Yong. Self-assembled liquid crystalline nanoparticles as a novel ophthalmic delivery system for dexamethasone: improving preocular retention and ocular bioavailability. Int J Pharm. 2010;396(1-2):179-87. doi: 10.1016/j.ijpharm.2010.06.015, PMID 20558263.

10. Kumar Singh Amit, Vivek Dubey, Vandana Arora. Role of natural polymer used in floating drug delivery system. J Pharm Sci Innov. 2012;1(3):11-5.

11. Hetangi Rathod, Vishnu Patel, Moin Modasia. In situ gel as a novel approach of gastro retentive drug delivery. Int J Pharm Life Sci. 2010;1(8):440-7.

12. Boyd. Characterization of drug release from cubosomes by using pressure ultrafiltration method. Int $\mathrm{J}$ Pharm 20003;260(2):239-47.

13. Geraghty PB, Attwood D, Collett JH, Sharma H, Dandiker Y. An investigation of the parameters influencing the bioadhesive properties of Myverol 18-99/water gels. Biomaterials. 1997;18(1):63-7. doi: 10.1016/s0142-9612(96)00087-7, PMID 9003899.

14. Garg Gopal, Saraf Shailendra, Saraf Swarnlata. Cubosomes: an overview. Biol Pharm Bull. 2007;30(2):350-3. doi: 10.1248/ bpb.30.350, PMID 17268078. 